DOI: $10.15593 / 2224-9982 / 2016.44 .04$

УДК 621.438

\author{
В.А. Назукин ${ }^{1,2}$, В.Г. Августинович ${ }^{1}$ \\ ${ }^{1}$ Пермский национальный исследовательский \\ политехнический университет, Пермь, Россия \\ ${ }^{2} \mathrm{OАO} \mathrm{«Авиадвигатель»,} \mathrm{Пермь,} \mathrm{Россия}$
}

\author{
СРАВНЕНИЕ РАЗЛИЧНЫХ ПОДХОДОВ \\ К МОДЕЛИРОВАНИЮ ТЕЧЕНИЯ В ПРЕМИКСЕРАХ \\ МАЛОЭМИССИОННЫХ КАМЕР СГОРАНИЯ
}

\begin{abstract}
В настоящее время благодаря развитию высокопроизводительных вычислительных систем и методов численного моделирования появилась возможность разработать новую методологию проектирования камер сгорания, предполагающую активное использование газодинамических расчетов и существенное сокращение экспериментальных работ. Представленное исследование направлено на разработку методики проведения расчетов аэродинамики фронтовых устройств малоэмиссионных камер сгорания наземных газотурбинных установок. Осуществлен анализ влияния различных параметров расчетной модели на общую картину течения, осредненные во времени поля (профили) скорости, структуру прецессирующего вихревого ядра и частоту возмущений в потоке. Были использованы полная и усеченная модели фронтового устройства; несколько расчетных сеток, отличающихся размерами элементов; различные подходы к описанию турбулентного течения, такие как решение осредненных по Рейнольдсу уравнений НавьеСтокса (RANS, URANS) с наиболее часто используемыми моделями турбулентности, моделирование отсоединенных вихрей (DES) и моделирование с адаптивными масштабами (SAS). Была установлена зависимость структуры прецессирующего вихревого ядра, прогнозируемая при URANS-моделировании, от начальных условий. Анализ результатов выполненных расчетов позволил сформулировать рекомендации для численного моделирования закрученных потоков в премиксерах.
\end{abstract}

Ключевые слова: газотурбинный двигатель, камера сгорания, премиксер, закрученные потоки, компьютерное моделирование.

\author{
V.A. Nazukin ${ }^{1,2}$, V.G. Avgustinovich ${ }^{1}$ \\ ${ }^{1}$ Perm National Research Polytechnic University, Perm, Russian Federation \\ ${ }^{2}$ OJSC "Aviadvigatel", Perm, Russian Federation
}

\title{
COMPARISON OF DIFFERENT APPROACHES TO SIMULATION OF FLOWFIELD INSIDE LOW EMISSION COMBUSTION CHAMBERS PREMIXERS
}

At present due to evolution of high-performance computing system and numerical modeling methods there is opportunity to develop new methodology for gas turbine combustion chambers design, assuming wide use of computation fluid dynamic and reduction of experimental investigations. The pre- 
sent work is dedicated to development the methodology for aerodynamics computations applied to low emission gas turbine combustion chamber premixers. An analysis of effect of different computational model parameters on the flow field, time-averaged velocity fields (profiles), precessing vortex core structure and frequencies of flow perturbations has been performed. Both full and cut premixer models; set of computational grids with different element sizes; different approaches to turbulence modeling such as Reynolds Averaged Navier-Stokes (RANS and URANS) equations with the common used turbulence models, Detached Eddy Simulation (DES) and Scale Adaptive Simulation (SAS) were used. It was revealed that initial conditions have significant influence on the results of URANS modeling in terms of prediction of precessing vortex core structure. The recommendations for numerical modeling of swirling flow inside premixers were developed according to analysis of computation results. dynamic.

Keywords: gas turbine, combustion chamber, premixer, swirling flows, computational fluid

\section{Введение}

В последние десятилетия во всем мире большое внимание уделяется экологии, периодически ужесточаются требования к вредным выбросам предприятий, двигателей транспортных средств и т.д. В настоящее время такие фирмы, как General Electric, Siemens, Alstom, Mitsubishi Heavy Industries предлагают на рынке газотурбинные установки (ГТУ) для выработки электроэнергии и механического привода с эмиссией $\mathrm{NO}_{x}$ и $\mathrm{CO}$ менее 50 мг/нм ${ }^{3}[1,2]$, в то время как ГТУ российских производителей не обеспечивают подобный уровень выбросов. Существующие малоэмиссионные камеры сгорания были созданы на базе результатов экспериментальных исследований. В настоящее время в связи с развитием вычислительной техники и программного обеспечения для численного моделирования газодинамических процессов появилась возможность разработать новую методологию проектирования камер сгорания, предполагающую активное использование верифицированных расчетных методик и отказ от натурного эксперимента до этапа испытаний опытного образца.

В процессе разработки бедных малоэмиссионных камер сгорания особое внимание следует уделять проектированию устройств предварительной подготовки топливовоздушной смеси (премиксеров), так как параметры смеси на входе в жаровую трубу оказывают существенное влияние на процесс горения, в том числе на выбросы вредных веществ. При разработке фронтовых устройств основные трудности связаны с обеспечением высокого качества перемешивания топливовоздушной смеси $[3,4]$, проскоком пламени и его стабилизацией в канале предварительного перемешивания [5,6], а также генерацией периодических когерентных вихревых структур, вызывающих возмущения в потоке, которые могут способствовать возникновению виброгорения и повреж- 
дению конструкции камеры сгорания [7]. Исходя из обозначенных проблем, расчетные методики, используемые для аэродинамического проектирования премиксеров, должны с высокой точностью прогнозировать, во-первых, поля скорости, чтобы была возможность исключить низкоскоростные или застойные регионы для снижения вероятности проскока пламени; во-вторых, пульсации скорости, так как перемешивание топлива с воздухом осуществляется главным образом за счет турбулентных пульсаций; и, в-третьих, процессы образования и распространения вихревых структур, поскольку необходимо точно знать, какие возмущения в потоке они вызывают и какое влияние оказывают на осредненную картину течения.

Основная цель данной статьи - анализ применения различных подходов к трехмерному численному моделированию динамики жидкости для прогнозирования характеристик закрученных потоков в премиксерах камер сгорания наземных газотурбинных установок и разработка требований к расчетным моделям для их дальнейшего использования при проектировании фронтовых устройств. Требуется определить, как различные параметры расчетной модели влияют на результаты расчета. Во-первых, необходимо рассмотреть возможность использования усеченной расчетной модели, в которой не будет моделироваться течение в каналах завихрителя, следовательно, не будет неравномерности, вносимой лопатками / стенками каналов. Вместо завихрителя в этом случае задается равномерная закрутка потока. Использование усеченной модели наиболее привлекательно с точки зрения определения частоты периодических возмущений, вносимых в поток вихревыми структурами. Это связано с тем, что разрешение спектра, получаемого с помощью быстрого преобразования Фурье, обратно пропорционально количеству точек, которые используются для его построения. При шаге по времени $1 \cdot 10^{-5}$ с потребуется выполнение нестационарного расчета длительностью 100000 итераций для получения спектра с шагом по частоте 1 Гц. Таким образом, усеченная модель позволит существенно сократить суммарное время расчета за счет снижения размеров расчетной сетки, следовательно, времени выполнения одной итерации. Во-вторых, необходимо определить влияние размеров расчетной сетки как на осредненные во времени характеристики течения, так и на точность моделирования вихревых структур. В-третьих, необходимо рассмотреть различные модели турбулентности 
и постановки расчета. С точки зрения экономии вычислительных ресурсов для нахождения осредненных характеристик течения привлекательным является подход, основанный на использовании решения осредненных по Рейнольдсу уравнений Навье-Стокса (Reynolds Averaged Navier-Stokes - RANS), однако он не всегда позволяет получить хорошее совпадение с экспериментальными данными, особенно при моделировании течений с высокой закруткой или высокими градиентами параметров потока [8]. При моделировании вихревых структур интересным представляется сравнение нестационарного RANS (Unsteady RANS - URANS) с вихреразрешающими подходами, такими как моделирование отсоединенных вихрей (Detached Eddy Simulation - DES) и моделирование с адаптивными масштабами (Scale-Adaptive Simulation - SAS) с точки зрения затрачиваемых ресурсов и точности прогнозирования структуры вихрей и частоты возмущений, вызываемых ими в потоке. Наконец, необходимо определить влияние количества выполненных итераций на картину течения. Исходя из этого основные задачи работы были сформулированы следующим образом:

- построить несколько расчетных сеток для полной модели исследуемого премиксера, отличающихся размером элементов, а также расчетную сетку для усеченной модели;

- выполнить стационарные расчеты с различными моделями турбулентности, количеством итераций, используя расчетные сетки разного размера;

- выполнить нестационарные расчеты, используя различные исходные данные, сетки, модели турбулентности, количество итераций и шаг по времени;

- выполнить анализ результатов расчетов с целью оценки применимости различных подходов для моделирования течения сильно закрученных потоков в премиксерах малоэмиссионных камер сгорания.

\section{Экспериментальные данные}

В качестве основы для выполнения исследования был выбран эксперимент, проведенный в Университете Лоуборо (Великобритания), цель которого заключалась в исследовании распада вихря и предоставлении данных для верификации численных расчетов течения закрученных потоков. Описание экспериментальной установки и детальное 
описание условий испытаний и их результатов представлено в статьях [9-11], а также диссертациях [12, 13]. Исследуемый премиксер, представленный на рис. 1, включал в себя одиночный радиальный завихритель, канал предварительного перемешивания, а также центральный канал, через который подавалась незакрученная струя.

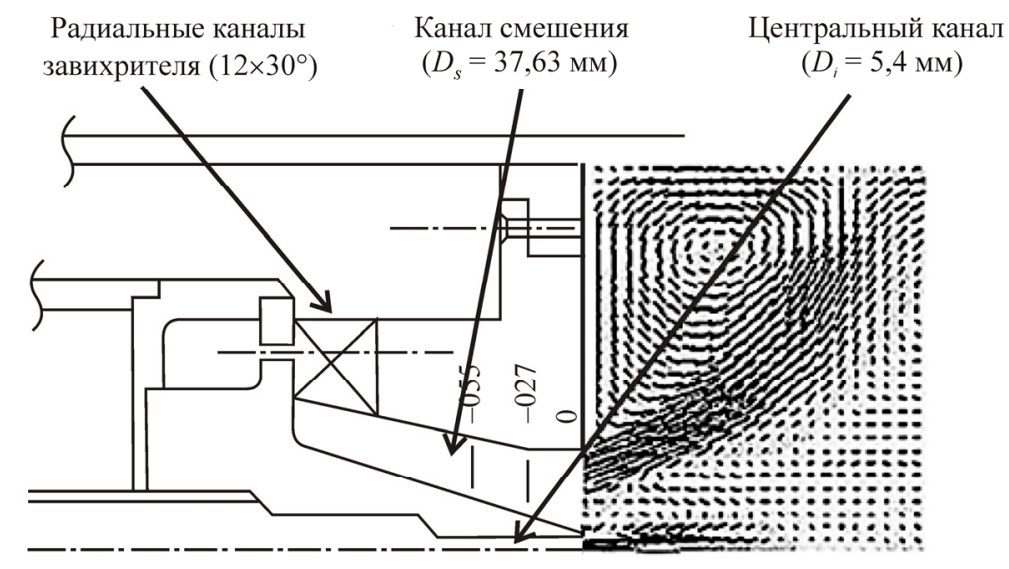

Рис. 1. Схема модельного премиксера

В ходе исследования были измерены как осредненные во времени, так и мгновенные поля скорости и пульсаций скорости, позволяющие получить информацию о структуре течения и вихревых структурах, генерируемых премиксером. Также были измерены пульсации осевой скорости в точке, лежащей ниже по потоку от выхода из канала предварительного перемешивания в области внутреннего сдвигового слоя. Были обнаружены пики на частотах 75 и 150 Гц, связанные с вращением двух сильных и двух более слабых вихревых структур.

Необходимо отметить, что при измерениях была использована вода, а не воздух, однако соблюдалось подобие по числам Рейнольдса с потоками в реальных конструкциях премиксеров. С точки зрения моделирования течения это существенно упрощает задачу поиска параметров модели, позволяющих прогнозировать частоту возмущений в потоке, поскольку из-за низких скоростей потока допускается использовать высокие значения шага по времени, следовательно, сокращается количество итераций, необходимых для построения спектров колебаний осевой скорости с разрешением по частоте не более 1-2 Гц. 


\section{Подготовка и выполнение расчетов}

Для выполнения расчетов было построено две модели: полная, включающая в себя каналы завихрителя, и усеченная, в которой вход в расчетную область располагался на уровне выхода из завихрителя. Для полной модели было построено три расчетные сетки (грубая, средняя и подробная), отличающиеся размером элементов, для усеченной - одна, соответствующая средней сетке для полной модели. Параметры расчетных сеток приведены в табл. 1. Во всех случаях для описания течения в пристеночном слое был построен призматический слой толщиной четыре элемента. Изображение средней расчетной сетки, а также области задания граничных условий представлено на рис. 2.

Таблица 1

\section{Параметры расчетных сеток}

\begin{tabular}{|l|c|c|c|c|}
\hline Постановка & $\begin{array}{c}\text { Обозначение } \\
\text { сетки }\end{array}$ & $\begin{array}{c}\text { Характерный } \\
\text { размер элементов } \\
\text { в премиксере, } \\
\text { мм }\end{array}$ & $\begin{array}{c}\text { Характерный } \\
\text { размер элементов } \\
\text { в жаровой трубе, } \\
\text { мм }\end{array}$ & $\begin{array}{c}\text { Общее } \\
\text { количество } \\
\text { элементов, } \\
\text { шт. }\end{array}$ \\
\hline Полная & $\# 1$ (Грубая) & 2 & 3,2 & 2566408 \\
\hline Полная & $\# 2$ (Средняя) & 1 & 2 & 7521088 \\
\hline Полная & $\# 3$ (Подробная) & 0,5 & 1,6 & 17215091 \\
\hline Усеченная & $\# 2 \_$сut & 1 & 2 & 5439535 \\
\hline
\end{tabular}

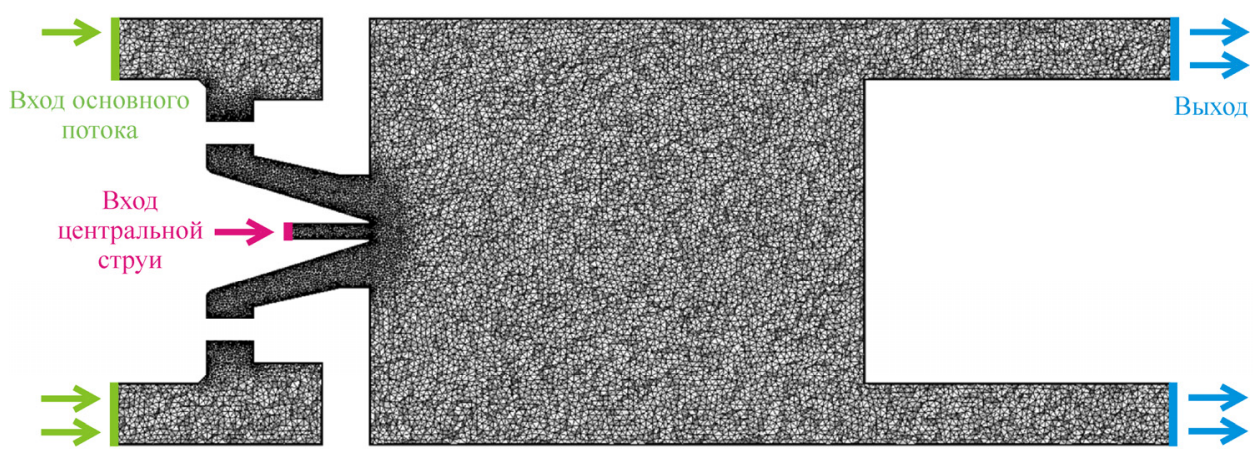

Рис. 2. Вид расчетной сетки \#2 
Для расчетов было использовано программное обеспечение ANSYS CFX v.14.5, в качестве граничных условий на входе задавались расходы воздуха (2,29142 кг/с для основного потока и 0,11176 кг/с для центральной струи) и температура потока $\left(15^{\circ} \mathrm{C}\right)$, а на выходе статическое давление 1 атм. При расчетах с усеченной моделью задавалось также направление потока в цилиндрических координатах под углом 59,5 $5^{\circ}$ к радиусу, который был определен при обработке результатов расчетов полной модели. При выполнении стационарных расчетов были использованы следующие модели турбулентности: $k-\varepsilon \mathrm{RNG}$, как наиболее простая и часто используемая при расчетах камер сгорания; SST (Shear Stress Transport), представляющая собой смесь $k-\varepsilon$ и $k-\omega$-моделей; модель напряжений Рейнольдса, а именно еe реализации SSG RSM, поскольку согласно электронному документу $^{1}$ она является наиболее подходящей для расчета закрученных потоков. Для нестационарных расчетов дополнительно были использованы вихреразрешающие DES- и SAS-модели турбулентности. Детальная информация обо всех моделях может быть найдена в приложении ${ }^{2}$. Метод моделирования крупных вихрей в чистом виде не рассматривался, так как его применение для анализа камер сгорания требует огромных вычислительных ресурсов и в настоящее время он не может быть использован для инженерных расчетов [14]. Шаг по времени в стационарных расчетах равнялся 0,001 с, а в большей части нестационарных - 0,0005 c, меньшие значения шага были использованы в расчетах с DES-моделью на мелкой сетке, чтобы внутри премиксера использовался метод крупных вихрей. Длительность каждого расчета указана в сводной таблице (табл. 2). В нестационарных расчетах с шагом по времени 0,0005 с последние 2000 итераций использовались для построения спектра пульсаций скорости, осреднение параметров потока происходило в течение 1000 итераций.

Для построения спектров пульсаций осевой скорости ее значение отслеживалось в той же точке, где производились измерения лазерным доплеровским анемометром в эксперименте, кроме того, отслежива-

\footnotetext{
${ }^{1}$ ANSYS CFX-Solver theory / ANSYS, Inc. 2010. Canonsburg, US.

${ }^{2}$ Там же.
} 
лась скорость в четырех точках, равномерно распределенных по окружности на среднем радиусе в сечении выхода из канала предварительного перемешивания.

Таблица 2

Параметры расчетов

\begin{tabular}{|c|c|c|c|c|c|c|}
\hline Номер & Тип расчета & Сетка & $\begin{array}{l}\text { Модель тур- } \\
\text { булентности }\end{array}$ & $\begin{array}{c}\text { Шаг по } \\
\text { времени }\end{array}$ & $\begin{array}{c}\text { Количество } \\
\text { итераций }\end{array}$ & $\begin{array}{c}\text { Начальные } \\
\text { условия }\end{array}$ \\
\hline 1 & Стационарный & $\# 1$ & $k-\varepsilon \mathrm{RNG}$ & $1 e-3$ & 1000 & - \\
\hline 2 & Стационарный & $\# 2$ & $k-\varepsilon \mathrm{RNG}$ & $1 \mathrm{e}-3$ & 1000 & - \\
\hline 3 & Стационарный & $\# 3$ & $k-\varepsilon \mathrm{RNG}$ & $1 e-3$ & 1000,2000 & - \\
\hline 4 & Стационарный & \#2_cut & $k-\varepsilon \mathrm{RNG}$ & $1 e-3$ & $\begin{array}{c}1000,2000 \\
4000\end{array}$ & - \\
\hline 5 & Стационарный & $\# 1$ & SST & $1 \mathrm{e}-3$ & 1000 & - \\
\hline 6 & Стационарный & $\# 2$ & SST & $1 \mathrm{e}-3$ & 1000 & - \\
\hline 7 & Стационарный & \#3 & SST & $1 e-3$ & 1000,2000 & - \\
\hline 8 & Стационарный & \#2_cut & SST & $1 e-3$ & $\begin{array}{c}1000,2000 \\
4000\end{array}$ & - \\
\hline 9 & Стационарный & $\# 1$ & SSG RSM & $1 e-3$ & 3000 & - \\
\hline 10 & Стационарный & $\# 2$ & SSG RSM & $1 e-3$ & 1000 & - \\
\hline 11 & Нестационарный & $\# 1$ & SST & $5 e-4$ & 2500 & № 5 \\
\hline 12 & Нестационарный & $\# 2$ & SST & $5 e-4$ & 2500 & № 6 \\
\hline 13 & Нестационарный & $\# 3$ & SST & $5 e-4$ & 2500 & № $7-1000$ \\
\hline 14 & Нестационарный & \#3 & SST & $5 e-4$ & 2500 & № $3-1000$ \\
\hline 15 & Нестационарный & \#2_cut & SST & $5 e-4$ & 2500 & № $8-1000$ \\
\hline 16 & Нестационарный & \#2_cut & SST & $5 e-4$ & 2500,10000 & № $4-2000$ \\
\hline 17 & Нестационарный & $\# 2$ & $k-\varepsilon \mathrm{RNG}$ & $5 e-4$ & 2500 & № 2 \\
\hline 18 & Нестационарный & $\# 2$ & SAS & $5 e-4$ & 2500,3500 & № 6 \\
\hline 19 & Нестационарный & $\# 1$ & DES & $5 e-4$ & 3000 & № 5 \\
\hline 20 & Нестационарный & $\# 2$ & DES & $5 e-4$ & 3000 & № 6 \\
\hline 21 & Нестационарный & \#3 & DES & $5 e-4$ & 3000 & № $7-2000$ \\
\hline 22 & Нестационарный & $\# 3$ & DES & $2 \mathrm{e}-4$ & 6000 & № $7-2000$ \\
\hline 23 & Нестационарный & \#2_cut & DES & $5 e-4$ & 3000 & № $8-1000$ \\
\hline 24 & Нестационарный & \#2_cut & DES & $5 e-4$ & 3000 & № $4-2000$ \\
\hline
\end{tabular}




\section{Анализ результатов}

Стационарные paсчеты. Для сравнения с экспериментом все скорости были обезразмерены относительно средней осевой скорости на выходе из премиксера, которая наблюдалась в эксперименте: $v_{x, s}=2,13 \mathrm{~m} / \mathrm{c}$, а размеры - относительно наружного диаметра канала предварительного перемешивания $D_{s}=37,63$ мм.

В связи с высокой закруткой потока и наличием прецессирующих вихревых структур в некоторых расчетах получалось квазистационарное решение: наблюдались значительные пульсации скорости на выходе из премиксера, зона обратных токов (ЗОТ) была существенно меньше, чем в эксперименте, и прецессировала вокруг оси премиксера. На рис. 3 представлены поля скорости, полученные в эксперименте, а также в расчетах № 6 и 10.

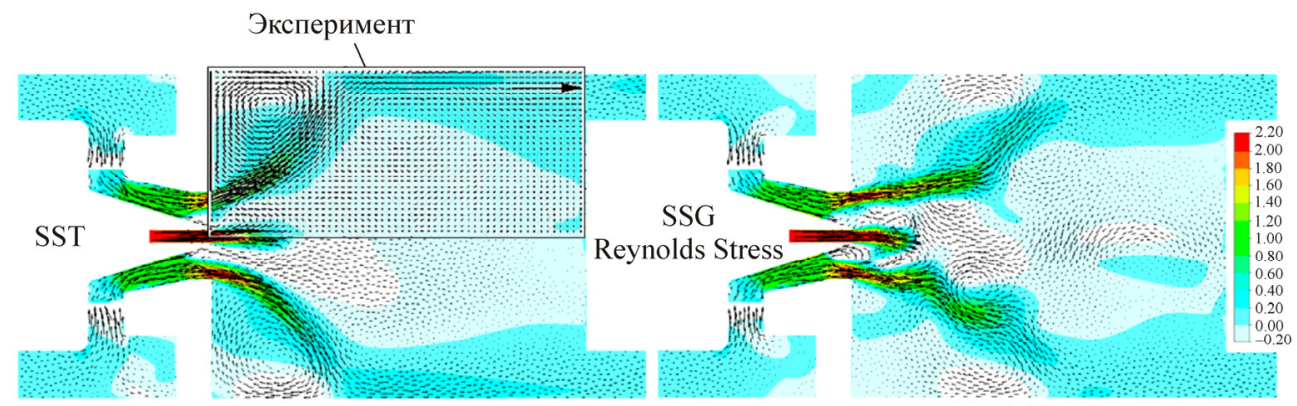

Рис. 3. Поля безразмерной осевой скорости в эксперименте и расчетах № 6 и 10

Было установлено, что в стационарных расчетах на стабильность расчета оказывают влияние расчетная сетка и модель турбулентности. Наиболее устойчивые результаты показала SST-модель турбулентности, она позволила получить устойчивое решение на всех расчетных сетках, однако на детальной сетке потребовалось увеличить время расчета для установления течения с 1000 до 2000 итераций. При использовании $k-\varepsilon$ RNG-модели устойчивое решение получено для грубой и средней расчетных сеток, на подробной сетке структура течения уже не соответствовала эксперименту и наблюдались существенные пульсации скорости. Модель напряжений Рейнольдса (SSG RSM) не позволила получить устойчивое решение ни на грубой, ни на средней (см. рис. 3) сетках. Исходя из этого наиболее подходящей для выполнения расчетов закрученных потоков является SST-модель турбулент- 
ности, так как она позволяет получить устойчивое стационарное решение даже при существенно нестационарном характере течения.

На рис. 4 представлены профили осевой и окружной скорости на выходе из канала предварительного перемешивания, а на рис. 5 - профили осевой скорости в различных сечениях, расположенных ниже по течению от выхода из премиксера.
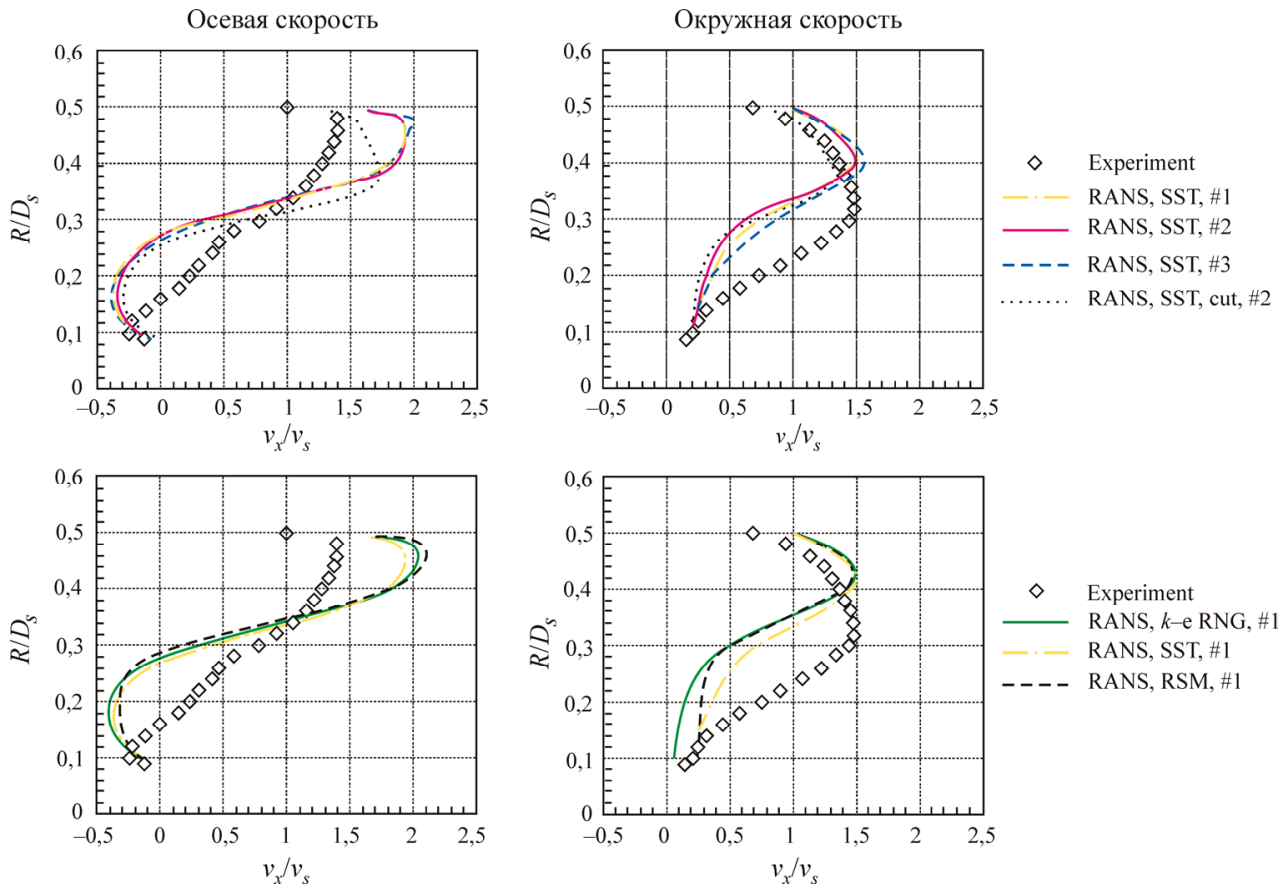

Рис. 4. Профили скорости на выходе из премиксера

Как видно из графиков на рис. 4, при расчетах с SST-моделью турбулентности отсутствует видимое влияние расчетной сетки на профили скорости на выходе из премиксера. Существенное влияние модели турбулентности также отсутствует. Во всех стационарных расчетах профили скорости на выходе из премиксера отличаются от экспериментальных, что связано с неправильным прогнозированием проникновения ЗОТ в канал предварительного перемешивания. В расчетах область, в которой наблюдается отрицательная осевая скорость, примерно в три раза больше, чем в эксперименте, из-за чего для сохранения расхода жидкости увеличивается осевая скорость в остальной части сечения (максимальное значение скорости на 30 \% больше, чем 

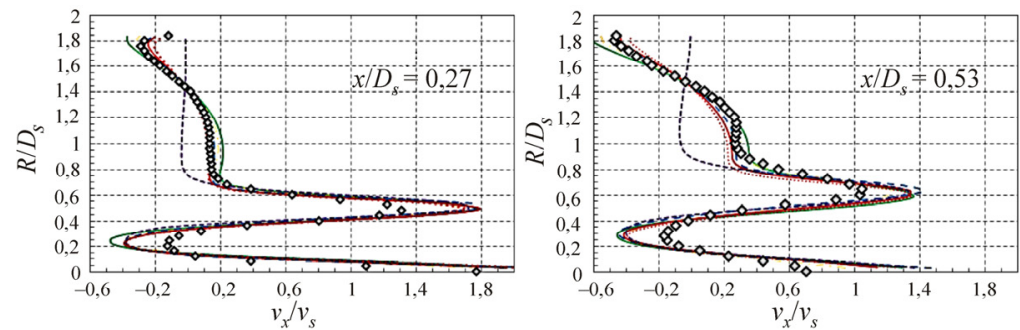

$\diamond$ Experiment

- RANS, $k$-e RNG, \#1
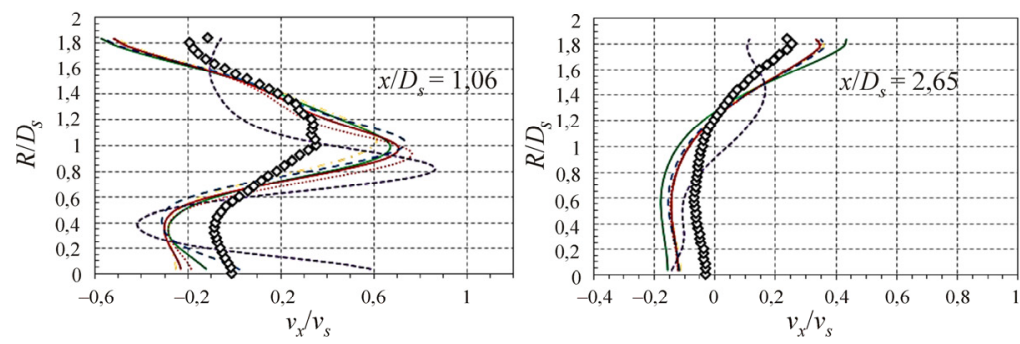

$=:=$ RANS, SST, \#1

- RANS, SST, \#2

-.-.. RANS, RSM, \#1

-.- RANS, SST, \#3

....... R RNSS, SST, cut, $\# 2$

Рис. 5. Профили осевой скорости в различных сечениях

в эксперименте) и неверно прогнозируется распределение окружной скорости.

При рассмотрении профилей осевой скорости в различных сечениях (см. рис. 5) можно увидеть, что во всех случаях, кроме использования модели SSG RSM, форма профиля скорости соответствует эксперименту, однако амплитуда ее изменения в расчетах больше, что, скорее всего, связано с высокой осевой скоростью на выходе из премиксера (в сечениях $x / D_{s}=0,27$ и $x / D_{s}=0,53$ скорость в кольцевой струе на 30 \% выше, чем в эксперименте). В расчетах с моделью SSG RSM профили скорости существенно отличаются от экспериментальных, что связано с неустойчивым характером течения. Стоит отметить, что на выходе из премиксера эта модель показала результаты, не отличающиеся от результатов других моделей турбулентности. Это говорит о том, что неверное прогнозирование распада вихря и структуры течения ниже по течению от выхода из премиксера не обязательно означает неверное прогнозирование течения внутри премиксера.

В данной задаче из-за того, что завихритель представляет собой каналы, расположенные под углом к радиусу, а толщина выходных кромок межканальных перегородок сопоставима с шириной каналов, после выхода из завихрителя происходит отклонение потока в сторону следов от перегородок, следовательно, увеличение крутки потока. Средний геометрический угол каналов по отношению к радиусу на вы- 
ходе из завихрителя составляет $54^{\circ}$, в то время как при обработке результатов расчетов на полной модели было установлено, что средний угол потока составляет 59,5 . Этот угол был использован для задания граничных условий при расчетах усеченной модели, которая с точки зрения полей скорости показала такие же результаты, как и полная, имеется лишь небольшое отличие в осевой скорости около наружного диаметра на выходе из премиксера. Распределение окружной скорости на выходе из завихрителя практически 1:1 совпадает с расчетом полной модели, что говорит о правильном определении крутки на входе.

В расчетах № 3, 4, 7 и 8 было выполнено различное количество итераций, результаты показали, что после установления течения количество шагов по времени не оказывает влияния на профили скорости и структуру течения. При этом было отмечено, что при увеличении количества итераций в стационарном расчете возникают колебания скорости, не оказывающие существенного влияния на течение в премиксере и ниже по течению. Причина этих колебаний заключается в возникновении прецессирующих вихревых структур. На рис. 6 представлены поля статического давления на выходе из премиксера, полученные в расчетах на полной и усеченной моделях с различными моделями турбулентности. Для полной модели показаны результаты расчетов на подробной сетке, поскольку на более грубых возмущениях они были значительно слабее.

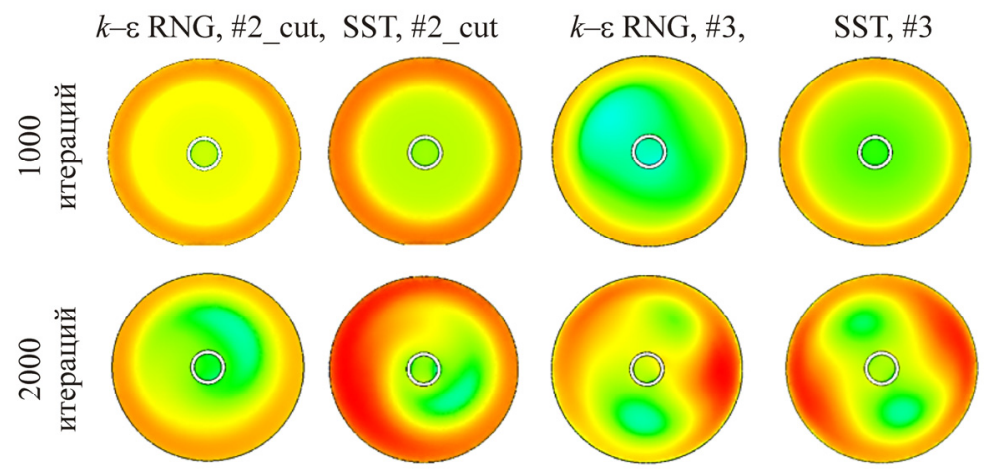

Рис. 6. Поля статического давления на выходе из премиксера

При расчетах с использованием полной модели наблюдается прецессирующее вихревое ядро (ПВЯ), состоящее из двух ветвей, в то время как на усеченной модели - из одной. При увеличении коли- 
чества итераций с 2000 до 4000 структура ПВЯ не изменялась. В дальнейшем эти стационарные расчеты были использованы для определения влияния начальных условий на результаты нестационарных расчетов.

В целом, несмотря на то что RANS-расчеты не обеспечивают высокой точности прогнозирования профилей скорости, они позволяют достаточно точно определить структуру течения в расчетной области и могут быть использованы для предварительной оценки аэродинамики премиксера.

Нестационарные расчеты. Выполненные нестационарные расчеты показали, что осредненная во времени структура течения хорошо согласуется с экспериментальной, исключение составляет величина проникновения центральной струи. Она увеличивается при уменьшении размера элементов расчетной сетки, особенно это проявляется в DES-расчетах, что представлено на рис. 7. При URANS-моделировании влияние не столь значительно.

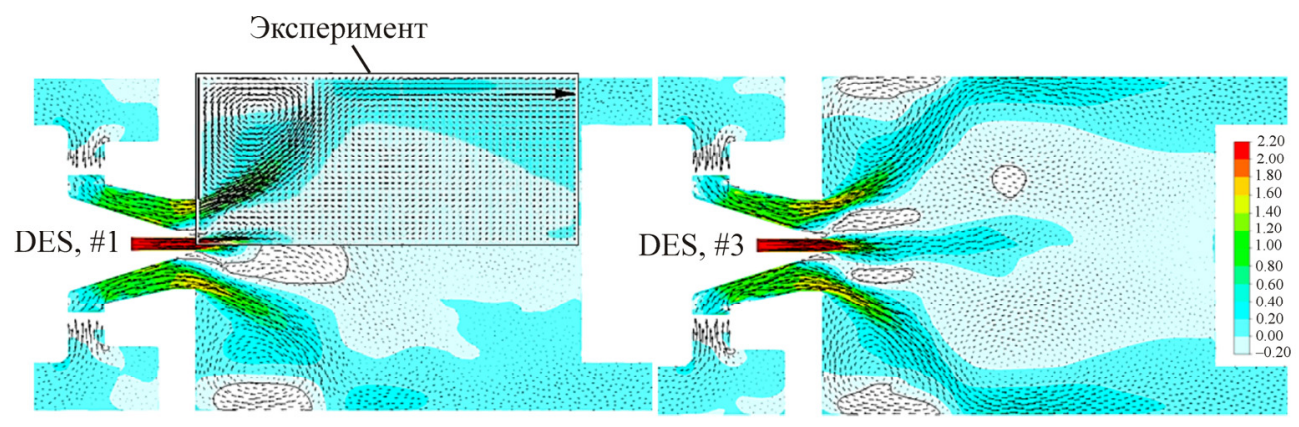

Рис. 7. Поля безразмерной осевой скорости в эксперименте и расчетах № 19 и 22

При сравнении расчетов, выполненных на различных расчетных сетках, было установлено, что ни при URANS-моделировании с SST-моделью турбулентности (№ 11-13), ни при DES-расчетах (№ 19-21) влияние размера элементов на осредненные во времени профили скорости не проявляется. Ниже представлены осредненные во времени профили скорости на выходе из премиксера (рис. 8) и осевая скорость в различных сечениях (рис. 9).

Так же как и в стационарных расчетах, модель турбулентности не оказывает существенного влияния на профили скорости на выходе из канала предварительного перемешивания и вблизи выхода из него, 

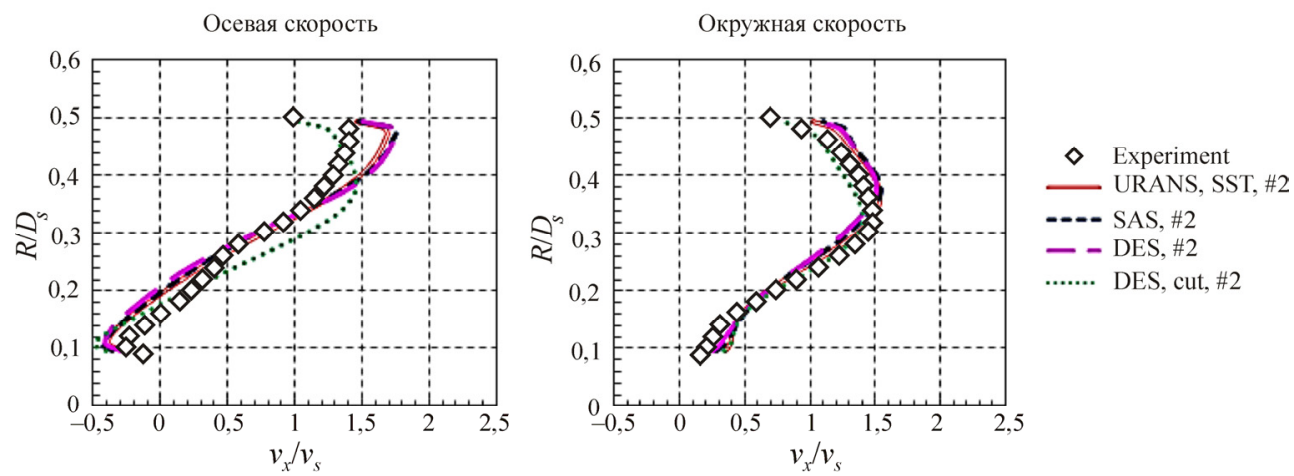

Рис. 8. Осредненные во времени профили скорости на выходе из премиксера
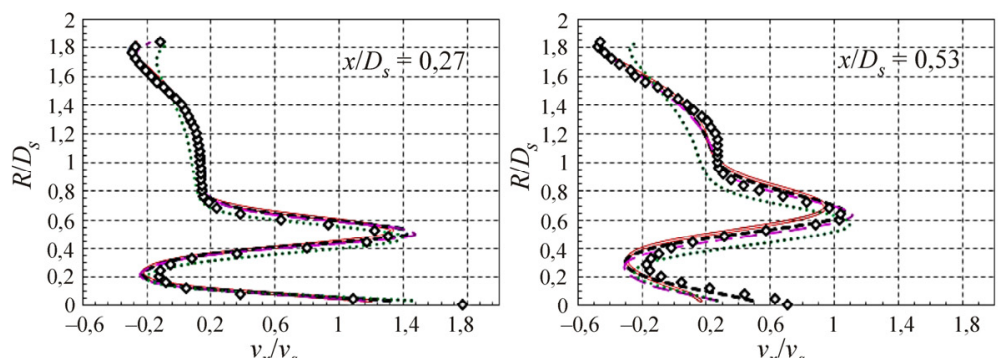

$\diamond$ Experiment

U URANS, SST, \#2
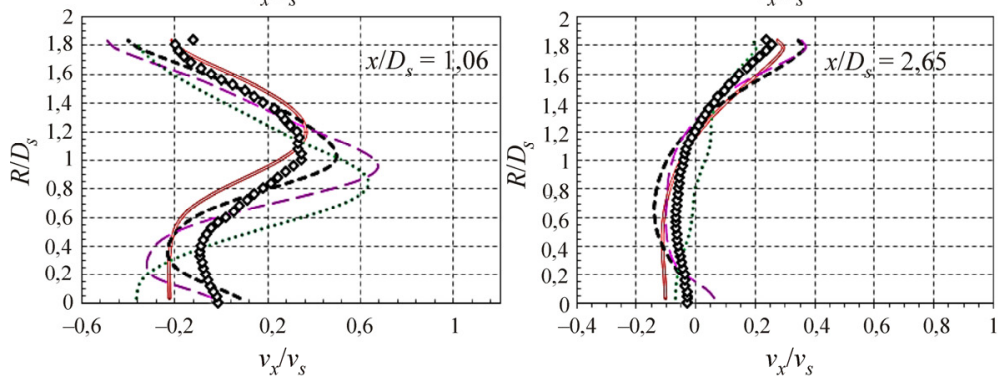

$--\cdot-$ SAS, $\# 2$

- DES, \#2

DES, cut, $H 2$

Рис. 9. Осредненные во времени профили скорости в различных сечениях

отличия проявляются только в сечениях $x / D_{s}=1,06$ и $x / D_{s}=2,65$. Нестационарные расчеты позволяют намного лучше предсказывать профили скорости, чем стационарные, в частности, точнее определяются величина проникновения 3ОТ внутрь премиксера и распределение окружной составляющей скорости. Более точное прогнозирование скорости на выходе из премиксера обеспечивает более точное прогнозирование скорости ниже по течению. При расчетах с использованием усеченной модели незначительно отличается профиль осевой скорости 
вблизи наружного диаметра, что, скорее всего, связано с отсутствием влияния следов от лопаток на обтекание угла при повороте потока после выхода из завихрителя.

При нестационарных расчетах большой интерес представляет влияние параметров расчетной модели на характеристики вихревых структур. В работах авторов эксперимента [11] было показано, что RANS-подход не позволяет смоделировать прецессирующее вихревое ядро, состоящее из двух сильных вихрей и двух слабых, следовательно, неверно прогнозируется частота возмущений, которые вносятся в поток вихревыми структурами. В данном исследовании подобная структура ПВЯ была воспроизведена во всех нестационарных расчетах, кроме № 16, в котором применялась SST-модель турбулентности, а в качестве начальных условий были использованы результаты стационарного расчета с прецессирующим вихревым ядром в виде одного вихря (см. рис. 6, $k-\varepsilon$ RNG, \#2_cut, 2000 итераций). В качестве проверки того, что сформировавшаяся структура течения неслучайна, длительность расчета была увеличена со стандартных 2500 шагов до 10 000. Также в процессе расчета искусственно создавались возмущения в виде колебания расхода через премиксер с амплитудой до $50 \%$, однако структура ПВЯ не изменилась. На рис. 10 показана схема, демонстрирующая влияние начальных условий на структуру ПВЯ в нестационарном расчете. В верхней части рисунка представлено поле статического давления на выходе из премиксера в стационарных расчетах, а в нижней - в нестационарных.
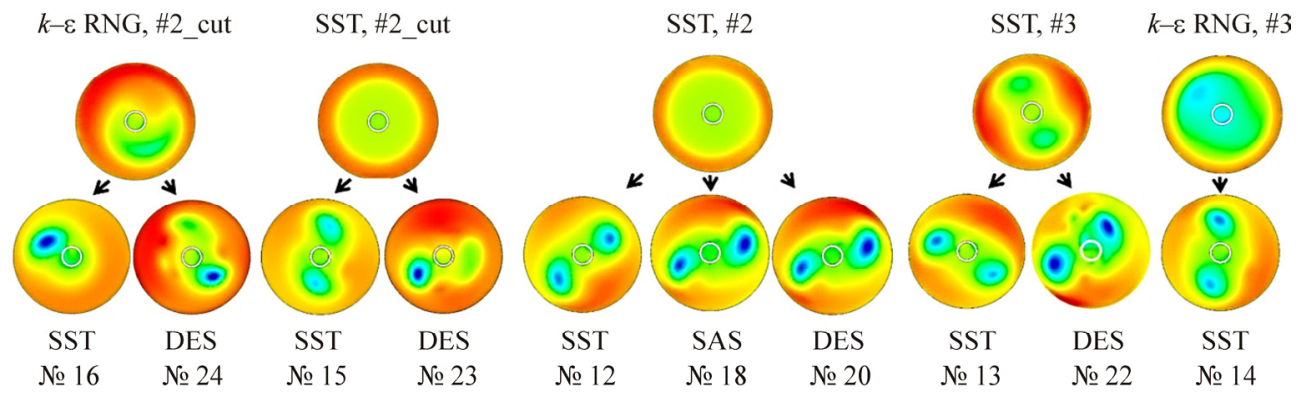

Рис. 10. Влияние начальных условий на структуру ПВЯ

Как видно из рис. 10, DES-модель турбулентности во всех случаях позволила корректно воспроизвести прецессирующее вихревое 
ядро, однако при расчетах на усеченной модели интенсивность вихрей и их взаимное угловое положение изменялись намного сильнее, чем в полной модели. SAS-модель позволила получить результаты, соответствующие эксперименту, но для более точной оценки ее способности прогнозировать вихревые структуры необходимо выполнить расчеты при различных начальных условиях. URANS-моделирование c SST-моделью турбулентности выявило зависимость результатов от начальных условий при использовании усеченной модели премиксера. При расчетах на полной модели структура ПВЯ при URANS-расчетах была спрогнозирована корректно, даже при начальных условиях, в которых ПВЯ состояло из одного вихря ( $k-\varepsilon$ RNG, \#3, 1000 итераций).

Правильное прогнозирование вихревых структур, генерируемых фронтовым устройством камеры сгорания, важно для того, чтобы, вопервых, иметь возможность оценить их влияние на структуру течения в камере сгорания, а во-вторых, определить частоту возмущений в потоке, поскольку колебания скорости и концентрации и связанные с ними колебания тепловыделения во фронте пламени способны привести к виброгорению. На рис. 11 представлены спектры мощности для осевой скорости в точке с координатами $x / D_{s}=0,27, r / D_{s}=0,27$. Во всех случаях частоты, связанные с прецессией вихревого ядра, оказалась незначительно завышены и лежат в диапазоне от 81 до 86 Гц для двух вихрей и от 163 до 172 - для четырех. На некоторых спектрах также присутствует пик на частоте, соответствующей вращению одного вихря. Это связано с тем, что один из вихрей вызывает более сильные возмущения в потоке. В экспериментах данного пика не наблюдалось, так что изменение интенсивности вихревых структур, возможно, является результатом численной неустойчивости. В качестве проверки было выполнено DES-моделирование на сетке \#3 с шагом по времени 0,000 01 с, но неустойчивость сохранилась. Несмотря на отсутствие данного пика в эксперименте, он не препятствует определению частоты прецессии ПВЯ, которая может быть установлена при сопоставлении спектра с изменением во времени поля статического давления на выходе из премиксера. Как для URANS-, так и для DESмоделирования характерно увеличение амплитуды шума при уменьшении размеров элементов расчетной сетки в связи с увеличением числа случайных возмущений в потоке, причем с DES-моделью уровень шума заметно выше. Это приводит к тому, что частоту, свя- 
занную с вращением четырех вихревых структур, становится сложно выделить на фоне других возмущений в потоке. SAS-модель показала спектр колебаний скорости с уровнем шума как при DES-расчетах, но в ней присутствует пик, соответствующий вращению одного вихря. Расчеты на усеченной модели позволили определить частоту прецессии вихревого ядра, но, как уже было показано, при URANS существует зависимость от начальных условий, а при DES наблюдались значительные колебания структуры ПВЯ, так что использование полной модели оказалось более предпочтительным. Интересен тот факт, что даже на грубых расчетных сетках удалось достаточно точно определить частоту вращения ПВЯ.
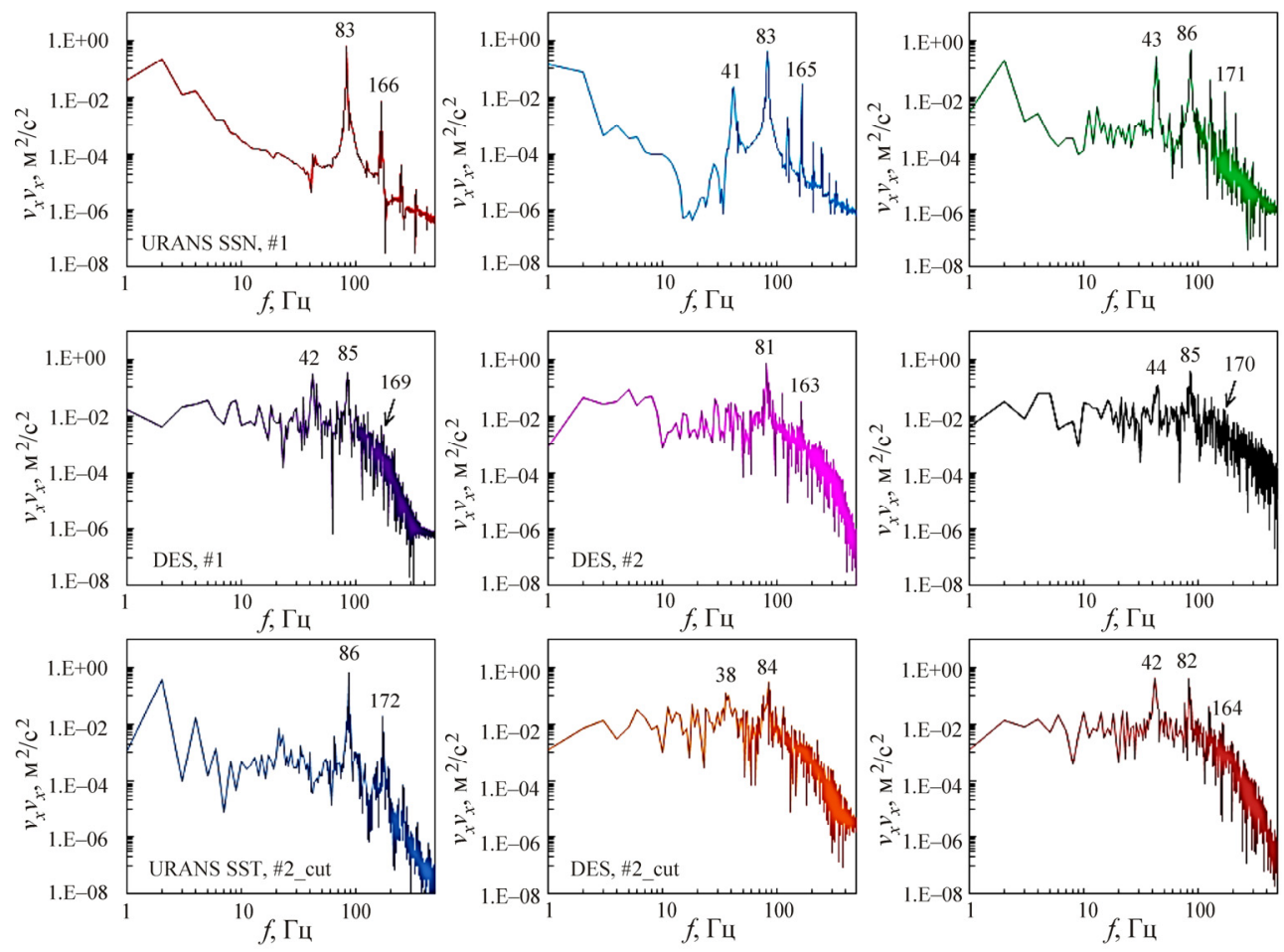

Рис. 11. Спектры мощности для осевой скорости в точке с координатами $x / D_{s}=0,27$,

$$
r / D_{s}=0,27
$$




\section{Заключение}

На основании выполненных расчетов могут быть сформулированы следующие рекомендации для моделирования закрученного потока во фронтовых устройствах камер сгорания:

1. Стационарные расчеты с SST-моделью турбулентности могут быть использованы для оценки структуры течения в расчетной области, определения формы и положения зон обратных токов и качественного определения полей скорости. Расчетная сетка не оказывает значительного влияния на результаты расчета, более того, использование подробной сетки может способствовать возникновению неустойчивого течения и появлению пульсаций параметров потока. В случае их возникновения расчет должен выполняться до установления квазистационарного течения.

2. Для детального определения структуры течения необходимо выполнять нестационарные расчеты. URANS-моделирование с SSTмоделью турбулентности, также как и DES-моделирование, позволяет с высокой точностью прогнозировать осредненные во времени поля скорости. Размер расчетной сетки, так же как и в стационарных расчетах, не влияет на профили скорости.

3. При необходимости моделирования вихревых структур, генерируемых фронтовым устройством, должны быть использованы DESподход и модель фронтового устройства с минимальным количеством упрощений. Несмотря на то, что использование грубых расчетных сеток позволяет определить структуру ПВЯ, использование более качественных сеток предпочтительнее, поскольку это позволяет моделировать не только ПВЯ, но и другие вихревые структуры, например следы от лопаток (каналов) завихрителя, что дает возможность анализировать их влияние на поток. Для определения частоты прецессии вихревого ядра может быть использована грубая сетка, чтобы сократить время выполнения расчета. Для определения частот более слабых вихрей требуется качественная сетка, но необходимо принимать во внимание тот факт, что с увеличением сеточного разрешения становится сложно выделить слабые периодические возмущения на фоне возрастающего по амплитуде шума.

4. Нестационарные расчеты с использованием усеченной модели позволяют определять поля скорости с такой же точностью, как с полной. Но следует учитывать тот факт, что угол крутки потока, который 
должен задаваться на входе в расчетную область, может отличаться от геометрического угла выходной кромки лопаток (каналов) завихрителя. В связи с этим усеченные модели премиксера целесообразно использовать для задач по определению влияния закрутки потока в завихрителе, формы (размеров) канала предварительного перемешивания или формы (размеров) жаровой трубы на осредненные во времени характеристики течения, поскольку, во-первых, упрощается процесс построения расчетной сетки (при расчетах с различной закруткой вообще отсутствует необходимость перестроения сетки), а во-вторых, сокращается время выполнения каждого расчета.

Дальнейшее исследование будет направлено на проверку разработанных рекомендаций при проведении расчетов течения сжимаемой жидкости, а также на разработку методики для точного прогнозирования полей концентрации.

\section{Библиографический список}

1. Technology update on gas turbine dual fuel, dry low emission combustion systems / P.E. Rokke, J.E. Husgand, N.A. Rokke, O.N. Svendsgaard // Proceedings of ASME Turbo Expo, 2003, June 16-19, Atlanta. Paper № GT2003-38112.

2. Новейшие разработки технологии DLN для турбин класса F: DLN 2.6+ / К. Венкатараман, C.Е. Льюис, Дж. Натарайан, С.P. Томас, Дж.В. Ситено // Газотурбинные технологии. - 2012. - № 1(102). C. 26-33.

3. Modern gas turbine systems / ed. P. Jansohn. - Cambridge: Woodhead Publishing Ltd., 2013. - 816 p.

4. Barnes J.C., Mellor A.M. Quantifying unmixedness in lean premixed combustors operating at high pressure, fired conditions // Proceedings of ASME 1997 International Gas Turbine and Aeroengine Congress and Exhibition, 1997, June 2-5, Orlando. - Paper № 97-GT-073.

5. Baumgartner G., Sattelmayer Th. Experimental investigation of the flashback limits and flame propagation mechanisms for premixed hydrogenair flames in non-swirling and swirling flow // Proceedings of ASME Turbo Expo, 2013, June 3-7, San Antonio. - Paper № GT2013-94258.

6. Fritz J., Kroner M., Sattelmayer Th. Flashback in a swirl burner with cylindrical premixing zone // ASME Turbo Expo, 2001, June 4-7, New Orleans. - Paper № 2001-GT-0054. 
7. Gupta A.K., Lilley D.G., Syred N. Swirling flows. - Tunbridge Wells: Abacus Press, 1984. - 475 p.

8. Волков К.Н., Емельянов В.Н. Моделирование крупных вихрей в расчетах турбулентных течений. - М.: Физматлит, 2008. - 368 с.

9. Midgley K., Spencer A., McGuirk J.J. Unsteady flow structures in radial swirler fed fuel injectors // Journal of Engineering for Gas Turbines and Power. - 2004. - Vol. 127(4). - P. 755-764.

10. Spencer A., McGuirk J.J., Midgley K. Vortex breakdown in swirling fuel injector flows // Journal of Engineering for Gas Turbines and Power. - 2008. - Vol. 130(2).

11. Comparison of URANS and LES CFD methodologies for air swirl fuel injectors / D. Dunham, A. Spencer, J.J. McGuirk, M. Dianat // Journal of Engineering for Gas Turbines and Power. - 2008. - Vol. 131(1).

12. Midgley K. An isothermal experimental study of the unsteady fluid mechanics of gas turbine fuel injector flowfields. Ph. D. thesis / Loughborough University. - Loughborugh, 2005. - 257 p.

13. Dunham D. Unsteady fluid mechanics of annular swirling shear layers. Ph. D. thesis / Loughborough University. - Loughborugh, 2011. $297 \mathrm{p}$.

14. Agarwal K.K., Konakalla S.R., Selvan S. On meshing guidelines for large eddy simulations of gas turbine combustors // ASME Turbo Expo, 2014, June 16-20, Düsseldorf. - Paper № GT2014-26077

\section{References}

1. Rokke P.E., Husgand J.E., Rokke N.A., Svendsgaard O.N. Technology update on gas turbine dual fuel, dry low emission combustion systems. Proceedings of ASME Turbo Expo, 2003, June 16-19, Atlanta. Paper no. GT2003-38112.

2. Venkatamaran K., Lewis S.E., Natarajan G., Thomas S.R., Siteno G.V. Noveyshie razrabotki tekhnologii DLN dlya turbin klassa F: DLN 2.6+ [The newest developments of DLN technology applied for F-class gas turbines: DLN 2.6+]. Gazoturbinnie tehnologii, 2012, no. 1(102), pp. 26-33.

3. Modern gas turbine systems. Ed. P. Jansohn. Cambridge: Woodhead Publishing Ltd., 2013. 816 p.

4. Barnes J.C., Mellor A.M., Quantifying unmixedness in lean premixed combustors operating at high pressure, fired conditions. Proceedings 
of ASME 1997 International Gas Turbine and Aeroengine Congress and Exhibition, 1997, June 2-5, Orlando. Paper no. 97-GT-073.

5. Baumgartner G., Sattelmayer Th. Experimental investigation of the flashback limits and flame propagation mechanisms for premixed hydrogen-air flames in non-swirling and swirling flow. Proceedings of ASME Turbo Expo, 2013, June 3-7, San Antonio. Paper no. GT2013-94258.

6. Fritz J., Kroner M., Sattelmayer Th. Flashback in a swirl burner with cylindrical premixing zone. ASME Turbo Expo, 2001, June 4-7, New Orleans. Paper No. 2001-GT-0054.

7. Gupta A.K., Lilley D.G., Syred N. Swirling flows. Tunbridge Wells: Abacus Press. 1984. 475 p.

8. Volkov K.N., Emelyanov V.N. Modelirovanie krupykh vikhrey v rashchyotakh turbulentnykh techeniy [Large eddy simulation of turbulent flows]. Moscow: Phismatlit, 2008. 368 p.

9. Midgley K., Spencer A., McGuirk J.J. Unsteady flow structures in radial swirler fed fuel injectors. Journal of Engineering for Gas Turbines and Power, 2004, vol. 127(4), pp. 755-764.

10. Spencer A., McGuirk J.J., Midgley K. Vortex breakdown in swirling fuel injector flows. Journal of Engineering for Gas Turbines and Power, 2008, vol. 130(2).

11. Dunham D., Spencer A., McGuirk J.J., Dianat M. Comparison of URANS and LES CFD methodologies for air swirl fuel injectors. Journal of Engineering for Gas Turbines and Power, 2008, vol. 131(1).

12. Midgley K. An isothermal experimental study of the unsteady fluid mechanics of gas turbine fuel injector flowfields. Ph. D. thesis. Loughborough University, 2005. 257 p.

13. Dunham D. Unsteady fluid mechanics of annular swirling shear layers. Ph. D. thesis. Loughborough University, 2011. 297 p.

14. Agarwal K.K., Konakalla S.R., Selvan S. On meshing guidelines for large eddy simulations of gas turbine combustors. ASME Turbo Expo, 2014, June 16-20, Düsseldorf. Paper no. GT2014-26077.

\section{Об авторах}

Назукин Владислав Алексеевич (Пермь, Россия) - аспирант кафедры «Авиационные двигатели» ФГБОУ ВПО ПНИПУ (614990, г. Пермь, Комсомольский пр., д. 29); инженер-конструктор-расчетчик 
отдела камер сгорания ОАО «Авиадвигатель» (614990, г. Пермь, Комсомольский пр., д. 93, e-mail: v.a.naz@ narod.ru).

Августинович Валерий Георгиевич (Пермь, Россия) - доктор технических наук, профессор кафедры «Авиационные двигатели» ФГБОУ ВПО ПНИПУ (614990, г. Пермь, Комсомольский пр., д. 29, e-mail: august@avid.ru).

\section{About the authors}

Vladislav A. Nazukin (Perm, Russian Federation) - Postgraduate Student, Aviation Engines Department, Perm National Research Polytechnic University (29, Komsomolsky av., Perm, 614990); Simulation and Design Engineer, Combustor Chambers Department, OJSC “Aviadvigatel" (93, Komsomolsky av., 614990, e-mail: v.a.naz@ narod.ru).

Valeriy G. Avgustinovich (Perm, Russian Federation) - Doctor of Technical Science, Professor, Aviation Engines Department, Perm National Research Polytechnic University (29, Komsomolsky av., Perm, 614990, e-mail: august@avid.ru).

Получено 28.12.2015 